\title{
基于课程学习思想的目标检测增强算法
}

\author{
戴立伟，黄山 \\ (四川大学电气工程学院 成都 610065) \\ (3376321599@qq.com)
}

\begin{abstract}
摘 要：目标检测算法性能优劣既依赖于数据集样本分布，又依赖于特征提取网络设计. 从这 2 点出发，首先通过分 析 COCO 2017 数据集各尺度目标属性分布, 探索了数据集固有的导致小目标检测准确率偏低的潜在因素，据此提出 $\mathrm{CP}$ 模块, 该模块以离线方式调整数据集小目标分布，一方面对包含小目标图片进行上采样，另一方面对图片内小目 标进行复制粘贴. 然后, 针对网络特征提取能力问题, 受课程学习 $(\mathrm{CL})$ 思想启发, 提出 CL 层, 该层用目标标签引导 网络学习，用 CL 因子控制学习强度，使样本特征增强，便于网络进行特征提取. 在 COCO 2017 数据集上使用 CP 模 块, 并在 CenterNet 中嵌人 CL 层, 进行多组对比实验, 采用平均检测准确率、小目标检测准确率、中目标检测准确 率和大目标检测准确率作为评价指标, 实验结果证明了 CP 模块和 CL 层的有效性.
\end{abstract}

关键词: 课程学习; 目标检测; 特征提取

中图法分类号: TP391.41 DOI: 10.3724/SP.J.1089.2021.18401

\section{Object Detection Enhancement Algorithm Based on Curriculum Learning}

Dai Liwei and Huang Shan

(College of Electrical Engineering, Sichuan University, Chengdu 610065)

\begin{abstract}
The performance of object detection algorithms depends on both dataset distribution and network design of feature extraction. Starting from these two points, we firstly explore the potential inherent reasons that lead to low detection accuracy of small object by analyzing the distribution of object attributes at various scales in the COCO 2017 dataset, and propose copy and paste (CP) module accordingly, which adjusts the distribution of small object offline, on the one hand, upsampling the pictures containing small objects, on the other hand, copying and pasting the small objects in the pictures. Then, to further improve network feature extraction ability, inspired by the idea of curriculum learning (CL), we propose CL layer, which uses ground truth labels to guide the learning process, and CL factor to control the learning intensity, the features of objects are enhanced to facilitate network feature extraction. We deploy the CP module on the COCO 2017 dataset and embed the CL layer in the CenterNet network to conduct multiple sets of comparative experiments, and use average detection accuracy, small object detection accuracy, medium object detection accuracy, and large object detection accuracy as evaluation indicators. The experimental results prove the effectiveness of CP module and CL layer.
\end{abstract}

Key words: curriculum learning; object detection; feature extraction

收稿日期: 2020-05-24; 修回日期: 2020-08-03. 戴立伟(1991一), 男, 硕士研究生, 主要研究方向为深度学习、机器视觉、目标检 测; 黄山(1969-), 男, 博士, 教授, 博士生导师, 主要研究方向为图像处理、智能交通. 
随着目标检测算法不断创新与进步，检测准

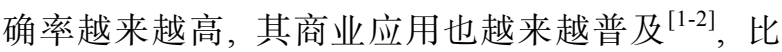
如人脸检测、车辆与行人检测 ${ }^{[3]}$ 、牲畜检测等需求 已基本满足. 但是, 当前基于深度学习的目标检测 算法仍存在 2 个普遍性问题: (1) 准确率依然有待 提升，特别是小尺度目标准确率偏低，这是影响目 标检测整体准确率的关键因素; (2) 网络特征提取 能力依然有待加强, 广义上讲, 特征提取是任何检 测网络的重要环节, 网络性能与其特征提取能力 息息相关

本文主要研究 One Stage 目标检测算法. One Stage 与 Two Stage 目标检测算法的区别是前者目 标分类准确率较高，而后者目标定位准确率较高， 目前 One Stage 与 Two Stage 算法准确率指标已不 存在明显差距. 早期 One Stage 算法以 SSD(single shot multibox detector $)^{[4]}$, RetinaNet $^{[5]}$ 和 YOLO(you only look once)系列算法 ${ }^{[6-8]}$ 为代表, SSD 利用 Anchor 机制，使用 6 个不同尺度特征层形成预测 金字塔(prediction pyramid，PP)进行预测，由于各 特征层分辨率不够高，导致小尺度目标检测准确 率较低. 与 SSD 类似, YOLOv3 利用网络最后 3 个 特征层分别预测大尺度、中尺度和小尺度目标，每 3 个 Anchor 为一组, 共 3 组分布在这 3 层上, 相比 SSD, YOLOv3 检测准确率提升明显, 但小尺度目 标准确率低的问题依然存在. RetinaNet 采用特征 金字塔网络 (feature pyramid networks, FPN $)^{[9]}$ 为特 征提取网络, 也在 3 种不同尺度特征层上进行预 测，其对小尺度目标检测能力优于 SSD 算法和 YOLO 系列算法, 且整体检测准确率提升明显. 不 过, 从这 3 个算法在 $\mathrm{COCO} 2017$ 数据集 ${ }^{[10]}$ 上的实 验结果可知，它们的整体检测准确率依然有待提 高, 特别是小尺度目标检测准确率较低. 这些检测 算法过度依赖特征层尺度, 暴露了特征提取能力 不足的缺点.

研究指出, Anchor 的存在是导致 One Stage 算 法产生正负样本不平衡的原因之一，降低了网络 训练速度且引入了超参数. 近年来, 针对目标检测 出现了 Anchor Free 算法, 其中基于关键点的代表 性算法有 CornerNet ${ }^{[11]}$ 和 CenterNet ${ }^{[12]}$. 它们都采 用 Hourglass ${ }^{[13]}$ 为特征提取网络，区别是 CornerNet 预测一对角点, 分别是目标左上角和右下角角点, 而 CenterNet 多预测一个中心区域的中心点，其特 征表达能力更强, 准确率比 CornerNet 更高. 基于 关键点的 Anchor Free 算法准确率比早期的 One Stage 算法有明显提高，原因在于采用了沙漏型特
征提取网络 Hourglass, 它以 FPN 思路为出发点进 行改良, 对各尺度目标有更好的兼容性, 将细粒度 的局部信息与粗粒度的全局信息融合起来. 但是, 这些算法在 COCO 2017 数据集上的实验结果显示 出小尺度目标检测准确率依然不高, 与中尺度和 大尺度目标准确率有很大差距.

为进一步缩小这种差距，同时提高网络的整 体检测准确率, 本文首先通过 COCO 2017 数据集 各尺度目标统计分布情况，分析了小尺度目标准 确率偏低的潜在因素，据此提出 CP(copy and paste) 模块, 其包含 CP-U(copy and paste-upsampling)和 CP-D(copy and paste-duplicating) 子模块，用离线方 式解决小尺度目标检测存在的问题. 此外, 为解决 网络特征提取能力不足的问题, 受课程学习 (curriculum learning, CL) ${ }^{[14]}$ 思想启发, 本文提出 $\mathrm{CL}$ 层, 在选定特征提取层上嵌人 CL 层能增强网 络特征提取能力. 本文采用 CenterNet, 在 $\mathrm{COCO}$ 2017 数据集上进行几组对比实验，验证方法的有 效性. CP 模块和 CL 层具有一般适用性, CP 模块不 仅可以在 COCO 2017 数据集上使用，也可用于其 他目标检测数据集, CL 层也不仅适用于 CenterNet, 同时适用于其他目标检测网络.

\section{$1 \mathrm{CP}$ 模块}

\section{$1.1 \mathrm{CP}$ 模块背景}

1.1.1 COCO 2017 数据集各尺度目标属性统计 COCO 2017 数据集目标按照面积(像素数)可 分为小尺度、中尺度和大尺度目标(下文简称小目 标、中目标和大目标), $32 \times 32$ 以下为小目标, $33 \times 33$ 至 $96 \times 96$ 为中目标, $97 \times 97$ 以上为大目标. 其训练 集包含 118287 张图片, 验证集包含 5000 张图片, 测试集包含 40670 张图片, 涵盖超过 80 个类别, 共有 860001 个目标标签(ground truth, GT). 训练 集各尺度目标属性统计如表 1 所示.

表 1 COCO 2017 训练集各尺度目标属性统计

\begin{tabular}{ccccc}
\hline $\begin{array}{c}\text { 目标 } \\
\text { 尺度 }\end{array}$ & $\begin{array}{c}\text { 目标数量 } \\
\text { 占比 } / \%\end{array}$ & $\begin{array}{c}\text { 图片数量 } \\
\text { 占比 } / \%\end{array}$ & $\begin{array}{c}\text { 目标面积 } \\
\text { 占比 } / \%\end{array}$ & $\begin{array}{c}\text { 平均 Anchor } \\
\text { 匹配数量 } / \text { 个 }\end{array}$ \\
\hline 小 & 41.4 & 51.8 & 1.2 & 1.0 \\
中 & 34.3 & 70.1 & 10.2 & 1.3 \\
大 & 24.3 & 82.3 & 88.6 & 2.5 \\
\hline
\end{tabular}

1.1.2 COCO 2017 训练集潜在问题分析

为结合表 1 统计结果进一步分析其训练集存 在的问题, 对比分析表 2 列出的主流 One Stage 算 法在 COCO 2017 数据集上的准确率指标 ${ }^{[15]}$, 其涵 
盖主流的 Anchor Based 和 Anchor Free 算法; 其 中, $\mathrm{AP}$ 为平均检测准确率 (average precision, $\mathrm{AP}$ ), $\mathrm{AP}_{\mathrm{S}}$ 为小目标检测准确率, $\mathrm{AP}_{\mathrm{M}}$ 为中目标检测准 确率, $\mathrm{AP}_{\mathrm{L}}$ 为大目标检测准确率. 可以看到, 这些 算法的 $\mathrm{AP}_{\mathrm{S}}$ 很低, 性能依然有很大提升空间.

从表 1 和表 2 的统计结果可以发现几点事实.

(1) 训练集小目标占比为 $41.4 \%$, 中目标和大 目标占比分别为 $34.3 \%$ 和 $24.3 \%$;
（2）训练集中图片仅有 $51.8 \%$ 的包含小目标，而 超过 $70.0 \%$ 的包含中目标, 超过 $80.0 \%$ 的包含大目标;

(3) 训练集小目标面积占比仅有 $1.2 \%$ ，相比 之下, 中目标面积占比是小目标的 8 倍以上, 达到 $10.2 \%$ ，大目标面积占比最大，达到 $88.6 \%$;

(4) 训练集小目标平均 Anchor 匹配数量仅有 1.0 个, 而中目标和大目标 Anchor 匹配数量分别为 1.3 和 2.5 个.

表 2 主流 One Stage 目标检测算法在 COCO 2017 数据集上的准确率 ${ }^{[15]}$

\begin{tabular}{|c|c|c|c|c|c|c|}
\hline \multicolumn{2}{|c|}{ 算法 } & 特征提取网络 & $\mathrm{AP} / \%$ & $\mathrm{AP}_{\mathrm{S}} / \%$ & $\mathrm{AP}_{\mathrm{M}} / \%$ & $\mathrm{AP}_{\mathrm{L}} / \%$ \\
\hline \multirow{6}{*}{ Anchor Based } & YOLOv2 & DarkNet19 & 21.6 & 5.0 & 22.4 & 35.5 \\
\hline & SSD513 & ResNet101 & 31.2 & 10.2 & 34.5 & 49.8 \\
\hline & DSSD513 & ResNet101 & 33.2 & 13.0 & 35.4 & 51.1 \\
\hline & YOLOv3 & DarkNet53 & 33.0 & 18.3 & 25.4 & 41.9 \\
\hline & RetinaNet & ResNet101 & 39.1 & 21.8 & 42.7 & 50.2 \\
\hline & RefineDet & ResNet101 & $36.4 / 41.8$ & $16.6 / 25.6$ & $39.9 / 45.1$ & $51.4 / 54.1$ \\
\hline \multirow{5}{*}{ Anchor Free } & CornerNet & Hourglass 104 & $40.5 / 42.1$ & $19.4 / 20.8$ & $42.7 / 44.8$ & $53.9 / 56.7$ \\
\hline & ExtremeNet & Hourglass 104 & $40.2 / 43.7$ & $20.4 / 24.1$ & $43.2 / 46.9$ & $53.1 / 57.6$ \\
\hline & FSAF & ResNeXt101 & $42.9 / 44.6$ & $26.6 / 29.7$ & $46.2 / 47.1$ & $52.7 / 54.6$ \\
\hline & CenterNet & Hourglass 52 & $41.6 / 43.5$ & $22.5 / 25.3$ & $43.1 / 45.3$ & $54.1 / 55.0$ \\
\hline & CenterNet & Hourglass 104 & $44.9 / 47.0$ & $25.6 / 28.9$ & $47.4 / 49.9$ & $57.4 / 58.9$ \\
\hline
\end{tabular}

注. 表中“””前后的数字分别表示单尺度准确率和多尺度准确率.

据此，可以分析影响小目标检测准确率的潜 在因素. 从(1)(2)可知, 训练集小目标数量多, 但 是包含小目标的图片数量少, 造成小目标数量与 对应图片数量分布不均衡, 这是导致小目标准确 率偏低的一个重要因素.从(3)可以看到, 小目标 面积占比太低, 导致网络训练时很难学习到小目 标特征，从而造成小目标误检或漏检，网络倾向于 学习中目标和大目标特征, 这影响了网络整体目 标检测能力. 由于小目标面积太小, Anchor 与 GT 的 交并比(intersection over union, IOU)偏低, 导致小目 标的 Anchor 位置不准确, 结合(4)可知, 小目标匹配 Anchor 数量很少, 这限制了网络目标定位能力.

\section{$1.2 \mathrm{CP}$ 模块原理}

为解决上面提到小目标存在的问题，本文提 出一种离线处理模块, 取名 $\mathrm{CP}$ 模块, 其包含 2 个 子模块 CP-U 和 CP-D.

\subsection{1 上采样子模块 $\mathrm{CP}-\mathrm{U}$}

设输人训练集为 $D_{\mathrm{IN}}, \mathrm{CP}-\mathrm{U}$ 只对包含小目标 的训练集图片进行上采样, 输出训练集为 $D_{\mathrm{CP}}$. 不 同训练集对应不同的目标分布, 故上采样系数也 不同, 本文通过实验来确定上采样系数. 在 $\mathrm{CP}-\mathrm{U}$
中, 训练集图片上采样本质上可以理解为对某些 图片进行复制, 然后根据上采样系数进行粘贴.

\subsection{2 复制粘贴子模块 CP-D}

$\mathrm{CP}-\mathrm{D}$ 以 $D_{\mathrm{CP}}$ 为输人训练集, 设训练集 $D_{\mathrm{CP}}$ 中图 片 $I$ 存在 4 个目标, 分别为小目标 $A$ 和 $B$ 、中目标 $C$ 和大目标 $D$. 以 $A$ 为例, 复制 $A$ 为 $A_{1}$ 和 $A_{2}$ (或更多 份), 尺度保持不变, 以 0.5 的概率对 $A_{1}$ 和 $A_{2}$ 进行水 平翻转(或不翻转). 在以 $A$ 的 GT 中心点为圆心且 2 倍 $A$ 的 GT 长边为半径的圆周上粘贴 $A_{1}$ 和 $A_{2}$, 确保 $A_{1}, A_{2}$ 和 $A$ 互相不重叠. 对 $B$ 进行相同操作, $C$ 和 $D$ 保持不变. 对每张包含小目标的图片进行类似图 片 $I$ 的处理, 最后输出训练集 $D_{\mathrm{OUT}}$, 作为目标检测 网络训练集. CP-D 子模块流程如图 1 所示.

\section{$1.3 \mathrm{CP}$ 模块分析与讨论}

\subsection{1 子模块优点分析}

(1) CP-U 通过上采样增加包含小目标的图片 数量, 将其数量占比提高至与中目标和大目标接 近的水平, 解决小目标数量与其图片数量分布不 均衡问题.

(2) CP-D 通过复制粘贴多份小目标，使小目 
标面积相应增加，在一定程度上弥补了小目标特 征难以被充分学习和特征欠表示的缺点; 水平翻
转能增加小目标多样性, CP-D 还会使每个小目标 匹配的 Anchor 数量相应增加, 如图 2 所示.
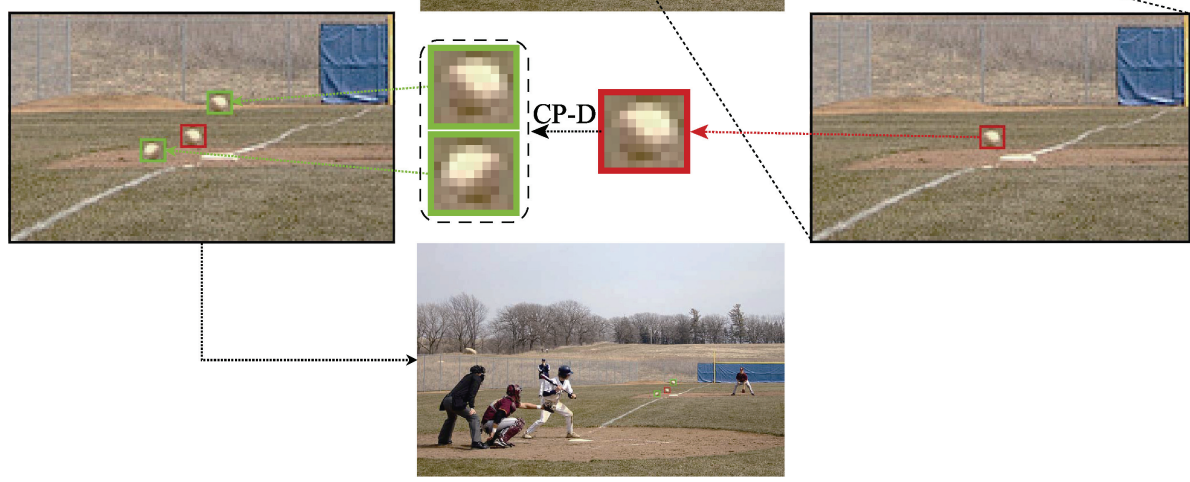

图 1 CP-D 子模块流程示意图

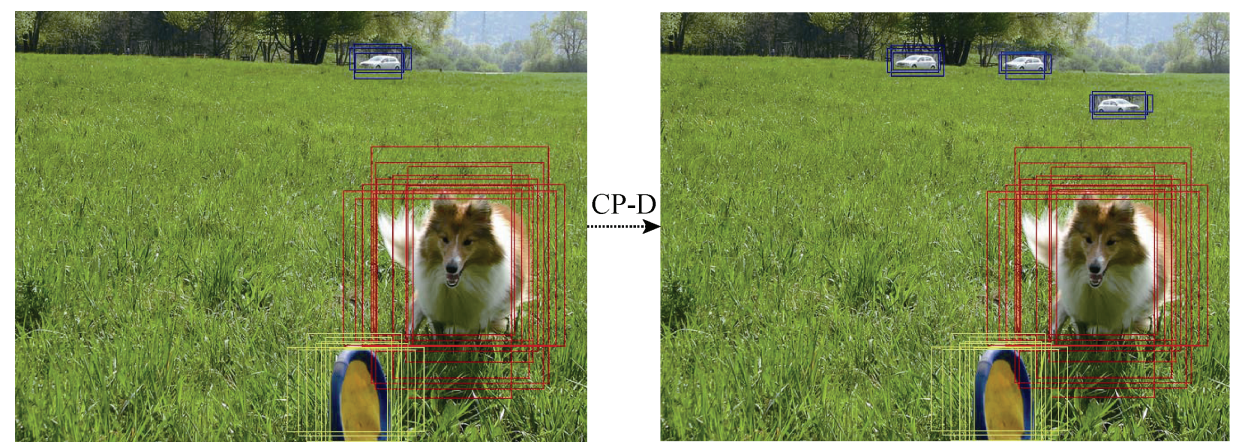

图 2 CP-D 子模块处理前后小尺度目标 Anchor 匹配数量变化对比图

\subsection{2 模块设计讨论}

(1) 在粘贴时, 之所以选择以小目标 GT 中心 点为圆心且 2 倍 $\mathrm{GT}$ 长边为半径的圆周上随机位置, 经验上来看，小目标周围更有可能存在相同尺度 的同类目标，如果随意在图片任意位置粘贴，可能 会破坏图片语义信息，导致语义信息不合理.

(2) 在粘贴时确保与原目标互相不重叠, 是为 了不影响小目标特征的完整性.

(3) 对小目标只进行水平翻转而不进行其他 操作如垂直翻转、旋转或放缩等, 是因为其他操作 某种程度上会破坏图片语义信息合理性，如放缩 会使小目标尺度发生变化, 脱离小目标定义; 垂直 翻转和大角度旋转小目标会导致图片缺乏可解释 性, 出现违背现实的情况, 这与旋转整张图片是不 同的. 水平翻转不破坏图片语义信息，既保证了小 目标尺度, 有利于后续分析对比实验, 又丰富了小 目标特征多样性, 有利于提高网络泛化能力.

\section{CL 层}

CL 思想解释了人类的学习是从简单到复杂的 一个逐步递进的过程, 学习先从容易的部分开始, 然后逐步过渡到困难的部分. 将 CL 思想融人目标 检测中, 让网络训练模仿人类学习方式, 能够使模 型找到更好的局部最优解, 同时加快训练速度和 收敛速度.

将本文提出的 CL 层嵌人到特征提取网络中, 增强网络特征提取能力. 实验中, 网络训练过程受 $\mathrm{CL}$ 因子 $\alpha$ 控制, $\alpha$ 越大, CL 影响越强; $\alpha$ 越小, $\mathrm{CL}$ 影响越弱, 训练是一个由浅人深的过程.

\subsection{CL 层原理介绍}

CL 层示意如图 3 所示.

CL 层算法流程如下:

Step1. 针对不同的目标检测网络, 在其特征提取 网络中选择合适的特征提取层 $F$. 


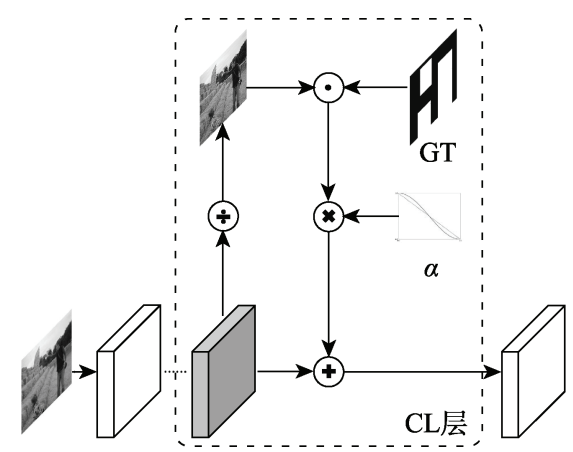

图 3 CL 层示意

Step2. 将 $F$ 所有特征图求均值, 输出一张均值特 征图 $F_{\mathrm{M}}$.

Step3. 获取输人图片的 GT, 构建 CL 模板, 即

$$
E_{(i, j)}=\left\{\begin{array}{ll}
1, & p_{(i, j)} \in \mathrm{GT} \\
0, & P_{(i, j)} \notin \mathrm{GT}
\end{array} .\right.
$$

其采用归一化, 将 GT 内像素值设为 1 , 将 GT 外像素值 设为 0 .

Step4. 将 $E$ 与 $F_{\mathrm{M}}$ 进行掩码运算, 输出 $F_{\mathrm{GT}}$. 掩码 运算有 2 种方式可供选择:

第 1 种是激活型, 采用 $F_{\mathrm{GT}}=E \cdot F_{\mathrm{M}}$ 将 $\mathrm{GT}$ 像素保留, 非 GT 像素归零, 在后续处理中能够达到将 GT 像素激 活而使非 GT 像素保持不变的效果;

第 2 种是抑制型, 采用 $F_{\mathrm{GT}}=-(1-E) \cdot F_{\mathrm{M}}$ 将 $\mathrm{GT}$ 像素 归零, 非 GT 像素取反, 在后续处理中会将非 GT 像素抑 制而使 GT 像素保持不变. 本质上激活型与抑制型是等 效的，但本文实验结果证明激活型表现比抑制型好.

Step5. 设计 CL 因子 $\alpha$. 本文设计了 2 种 $\alpha$ 因子, 曲线图如图 4 所示, 横轴代表训练进度 $t_{\mathrm{e}}$, 实线为非线 性 $\alpha$ 因子 $\alpha_{1}$, 虚线为线性 $\alpha$ 因子 $\alpha_{2}$, 它们分别为

$$
\alpha_{1}=0.5\left(1+\cos \left(t_{\mathrm{e}} \pi\right)\right) \text { 和 } \alpha_{2}=1-t_{\mathrm{e}} .
$$

其中, $t_{\mathrm{e}}=e_{\mathrm{C}} / e_{\mathrm{N}}$, 分子为当前迭代次数, 分母为总迭代

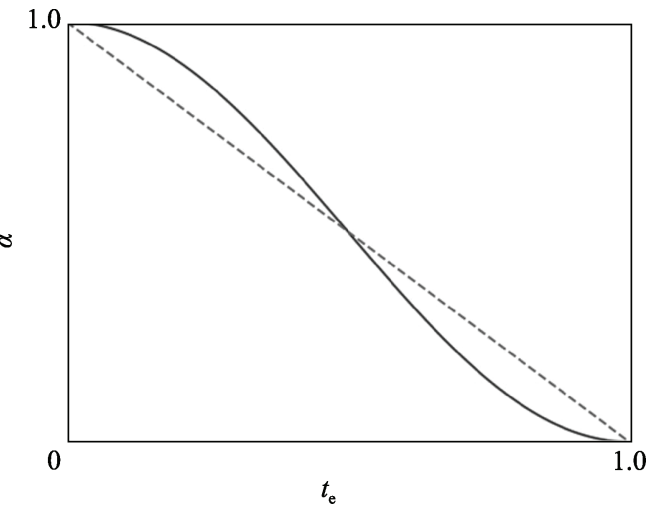

图 4 本文设计的 2 种 CL 因子曲线图
次数. 随着训练进行, $t_{\mathrm{e}}$ 由 0 逐渐变大, 最终为 1 ; 而 $\alpha$ 因子由 1 逐渐变小, 最终为 0 .

Step6. 将 $F_{\mathrm{GT}}$ 与 $\alpha$ 因子相乘, 输出 $F_{\alpha}$.

Step7. 将 $F_{\alpha}$ 与 $F$ 所有特征图逐通道相加, 最终获 得 $F_{\mathrm{CL}}$, 送给下一特征提取层.

以激活型为例, 训练过程采用非线性 $\alpha$ 因子 $\alpha_{1}$, 将 CL 层内部流程展开并可视化, 如图 5 所示.

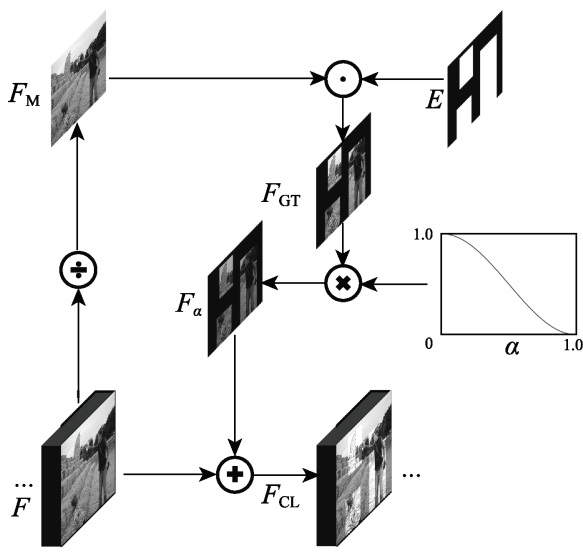

图 5 CL 层算法流程可视化示意图

\subsection{CL 层机制分析}

传统 CL 思想是先让模型学习容易的样本, 然 后逐渐加人复杂的样本进一步学习, 最后用全部 的样本再次训练. 相比之下, 本文注意到 GT 可以 起到引导学习的作用, 利用 GT 来引导网络从局部 范围开始学习，该局部范围包含目标特征，随着 $\alpha$ 因子的减小, 学习逐渐过渡到全局范围, 最后 GT 不再起作用. 从引导学习角度来说, 某种程度上 $\mathrm{CL}$ 层与注意力机制类似, 能够加快训练速度. 从 样本角度来说, CL 层会使正样本(前景)特征增强, 而使负样本(背景)特征减弱，使网络更容易进行目 标特征提取, 与此同时, CL 层会使网络关注正样 本, 加快网络训练收玫速度, 对网络的分类和定位 能力都有积极影响.

\subsection{CL 层嵌入方案}

\subsubsection{CenterNet 特征提取模块}

CenterNet 特征提取模块有 Hourglass52 和 Hourglass 104, 本文对比实验统一采用后者, 该模 块由 2 个 Hourglass52 模块叠加组成, 一共包含 104 层. 这种模块通过上采样和跳跃连接将局部特征 与全局特征融合在最后一层特征图上, 并在该特 征图上进行关键点检测. Hourglass 这种沙漏型特 征提取模块是 CenterNet 实现关键点检测的核心. 与传统 Hourglass 模块不同, CenterNet 有以下几点 改进: 
（1）在图片预处理模块中，图片先进行一次下 采样操作, 使用尺度为 $7 \times 7$ 、步长为 2 、深度为 128 的卷积操作进行, 然后接着一个步长为 2 、深度为 256 的残差模块.

(2) 下采样操作不再采用最大池化方式, 简单 地使用步长为 2 的卷积操作.

(3) 单个 Hourglass52 模块内进行 5 次下采样 操作, 各层深度对应为 $256,384,384,384$ 和 512 .

(4) 对特征层上采样操作前先经过 2 个残差模 块, 然后接着一个上采样操作, 该操作采用最近邻 插值方式. 应注意的是, 最中间深度为 512 的层采 用 4 个残差模块而不是 2 个.

（5）每个跳跃连接同样包含 2 个残差模块.

(6) 图片预处理模块的输出经过卷积和批归 一化操作后，与第 1 个 Hourglass52 模块的输出经 过卷积和批归一化操作后的结果进行跳跃连接, 之 后经过一个 ReLU(rectified linear units)激活函数层, 然后接一个残差模块, 最后送给第 2 个 Hourglass 52 模块.

(7) 将第 1 个 Hourglass52 模块的输出与第 2 个 Hourglass52 模块的输出进行跳跃连接, 获得最 后一层特征图，用来进行关键点检测.

\subsubsection{CL 层嵌人位置选择}

分析 CL 层机制可知, 嵌人时应选择拥有较 高分辨率且具有较丰富特征表示的特征层. 结合 Hourglass 104 模块的特殊结构, 本文将 CL 层嵌人 在 Hourglass 104 模块的第 1 个或第 2 个 Hourglass 52 模块最后一层特征层上，该层满足提到的条件. 以 嵌人第 1 个模块为例, 示意图如图 6 所示.

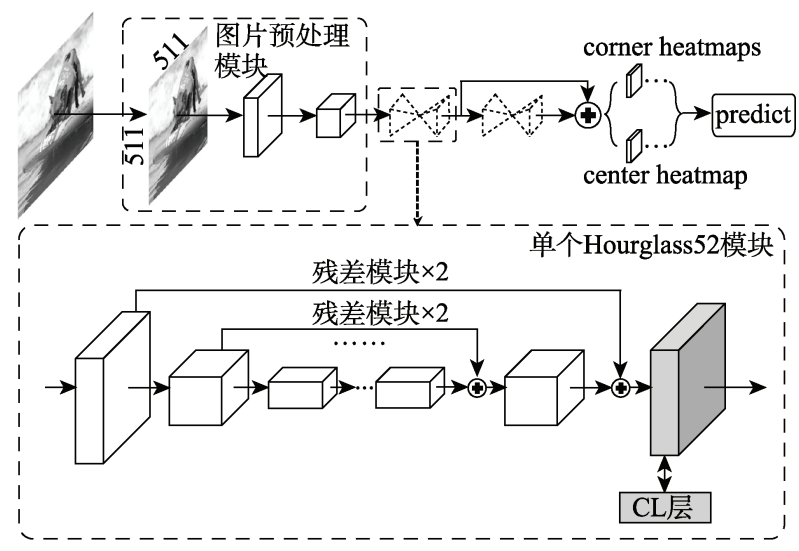

图 $6 \mathrm{CL}$ 层嵌人示意图

\section{3 实验与分析}

\section{1 实验环境}

本文软件框架为 64 位 Windows 10 家庭版操作
系统, CUDA v10.0.130 版本, CuDnn v7.5.0 版本, Python v3.7 版本, PyTorch v1.2.0 版本, OpenCV v3.4.2.16 版本, Torchvision v0.4.1 版本. 硬件配置 为 $2.6 \mathrm{GHz}$ Intel Core i7-9750H 6 核 $\mathrm{CPU}, 16 \mathrm{~GB}$ 内 存, $1365 \mathrm{MHz}$ NVIDIA GeForce RTX 2060 GPU, $6 \mathrm{~GB}$ 显存.

\section{2 评价指标}

本文实验都采用单尺度检测结果进行对比分 析, 每个实验结果以 5 次实验取平均值方式计算. 实验评价指标采用 $\mathrm{AP}, \mathrm{AP}_{\mathrm{S}}, \mathrm{AP}_{\mathrm{M}}$ 和 $\mathrm{AP}_{\mathrm{L}}$, 同时提 供模型训练时长和推理耗时数据.

\section{3 实验参数设置}

考虑实际硬件条件限制和训练时长因素, 为 使训练过程加速收玫并有效地获得评价指标, 本 文采用载人预训练模型方式进行对比实验，使用 已训练好的原 CenterNet 模型作为预训练模型. 本 文实验中, GPU 数量设置为 1 , Batch Size 设置为 2 , 在使用预训练模型的情况下, 总迭代次数设置为 600000 次，前 550000 次学习率设置为 0.00025 , 后 50000 次学习率设置为 0.000025 , 优化器选用 Adam, 其他参数保持与原 CenterNet 实验参数一致.

\section{4 对比实验与分析}

\section{4 .1 实验 1. CP-U 子模块实验}

为分析不同上采样系数对小目标检测准确率 的影响，设置不同的 CP-U 上采样系数进行对比实 验. 实验结果如表 3 所示. 其中, 第 1 行第 1 列对 应原 CenterNet 实验结果, 没有提供训练时长数据.

表 $3 \mathrm{CP}-\mathrm{U}$ 子模块不同上采样系数实验结果

\begin{tabular}{cccccc}
\hline 上采样系数 & $\mathrm{AP} / \%$ & $\mathrm{AP}_{\mathrm{S}} / \%$ & $\mathrm{AP}_{\mathrm{M}} / \%$ & $\mathrm{AP}_{\mathrm{L}} / \%$ & 训练时长 $/ \mathrm{h}$ \\
\hline & 44.9 & 25.6 & 47.4 & 57.4 & \\
2 & $\mathbf{4 5 . 1}$ & $\mathbf{2 6 . 3}$ & $\mathbf{4 7 . 4}$ & $\mathbf{5 7 . 3}$ & $\mathbf{1 6 1 . 2}$ \\
3 & 45.0 & 26.0 & 47.3 & 57.4 & 159.8 \\
4 & 44.7 & 25.7 & 47.2 & 57.1 & 160.7 \\
\hline
\end{tabular}

注. 粗体表示本实验最佳结果.

可以看到, 当上采样系数为 2 时, $\mathrm{AP}_{\mathrm{S}}$ 提升最 多, 并且 $\mathrm{AP}_{\mathrm{M}}$ 和 $\mathrm{AP}_{\mathrm{L}}$ 不受影响. 当上采样系数为 3 时, $\mathrm{AP}_{\mathrm{S}}$ 也有提升, 但 $\mathrm{AP}_{\mathrm{M}}$ 和 $\mathrm{AP}_{\mathrm{L}}$ 略有下降. 当上 采样系数为 4 时, $\mathrm{AP}_{\mathrm{S}}$ 提升有限, $\mathrm{AP}_{\mathrm{M}}$ 和 $\mathrm{AP}_{\mathrm{L}}$ 下降 明显, AP 反而低于原实验结果.

由实验结果可知, CenterNet 对 CP-U 子模块的 上采样系数是敏感的, 该系数不宜过高, 过高会带 来负面影响, 上采样系数需通过经验与实验来合 理选取. 


\subsection{2 实验 2. CP-D 子模块实验}

实验 2 在上采样系数为 2 的 CP-U 子模块基础 上进行, 对比小目标水平翻转与不翻转 2 种情况, 实验结果如表 4 所示. 其中，第 1 行第 1 列对应实 验 1 的最佳实验结果. 由实验结果可知, 不考虑翻
转, 采用粘贴数为 2 可使 $\mathrm{AP}_{\mathrm{S}}$ 明显提升, 而结合翻 转会得到本实验最佳结果. 随着粘贴数的增加, $\mathrm{AP}_{\mathrm{S}}$ 会逐渐下降, 粘贴数的选取对 $\mathrm{AP}_{\mathrm{S}}$ 的影响较为 明显, 而对 $\mathrm{AP}_{\mathrm{L}}$ 几乎没有影响, 对 $\mathrm{AP}_{\mathrm{M}}$ 的影响也 较小.

表 4 CP-D 子模块不同粘贴数实验结果

\begin{tabular}{|c|c|c|c|c|c|c|c|c|c|c|}
\hline \multirow{2}{*}{ 粘贴数 } & \multicolumn{5}{|c|}{ 不翻转 } & \multicolumn{5}{|c|}{ 水平翻转 } \\
\hline & $\mathrm{AP} / \%$ & $\mathrm{AP}_{\mathrm{S}} / \%$ & $\mathrm{AP}_{\mathrm{M}} / \%$ & $\mathrm{AP}_{\mathrm{L}} / \%$ & 训练时长/h & $\mathrm{AP} / \%$ & $\mathrm{AP}_{\mathrm{S}} / \%$ & $\mathrm{AP}_{\mathrm{M}} / \%$ & $\mathrm{AP}_{\mathrm{L}} / \%$ & 训练时长/ $\mathrm{h}$ \\
\hline & 45.1 & 26.3 & 47.4 & 57.3 & 161.2 & 45.1 & 26.3 & 47.4 & 57.3 & 161.2 \\
\hline 1 & 45.3 & 26.9 & 47.5 & 57.2 & 161.4 & 45.7 & 27.1 & 47.7 & 57.2 & 161.0 \\
\hline 2 & 45.7 & 27.2 & 47.8 & 57.2 & 160.7 & 45.9 & 27.3 & 47.9 & 57.1 & 161.1 \\
\hline 3 & 45.4 & 26.5 & 47.5 & 57.1 & 159.9 & 45.5 & 26.9 & 47.6 & 57.0 & 160.5 \\
\hline 4 & 45.2 & 26.4 & 47.4 & 57.3 & 161.1 & 45.2 & 26.5 & 47.3 & 57.1 & 161.3 \\
\hline
\end{tabular}

注. 粗体表示本实验最佳结果.

实验结果说明, CP-D 子模块小目标粘贴数应 适当选取，并不是越多越好. 从模型拟合角度来分 析，过多的重复性样本会造成网络训练过拟合，而 翻转操作会使样本具有多样性, 避免网络过拟合. 实验选取粘贴数为 2 是合适的，结合翻转操作进一 步提升了小目标检测准确率. 表 4 的实验结果表明 单独使用 $\mathrm{CP}$ 模块时, 评价指标 $\mathrm{AP}, \mathrm{AP}_{\mathrm{S}}$ 和 $\mathrm{AP}_{\mathrm{M}}$ 分 别提升了 $1.0 \%, 1.7 \%$ 和 $0.5 \%$, 而 $\mathrm{AP}_{\mathrm{L}}$ 仅下降了 $0.3 \%$.

实验 1 和实验 2 中使用了预训练模型，加快了 训练过程收敛速度, 迭代 600000 次, 训练时长约 为 $160 \mathrm{~h}$. 由于本文提出的 $\mathrm{CP}$ 模块以离线方式对数 据集进行处理，处理完毕后再进行网络训练，因此 该模块不影响训练时长.

\section{4 .3 实验 $3 . \mathrm{CL}$ 层实验}

实验分 3 部分进行. 第 1 部分不使用 $\mathrm{CP}$ 模块, 仅在 Hourglass 104 模块的第 1 个 Hourglass52 模块 最后一层特征层上嵌人 CL 层. 实验结果如表 5 所 示，其中第 1 列第 1 行对应原 CenterNet 实验结果, 推理耗时括号内外的数据分别是原 CenterNet 实验 结果和基于本文硬件条件的实验结果.

第 2 部分在嵌人 CL-layer 层的同时使用了 $\mathrm{CP}$ 模块, 其中 CP-U 上采样系数为 $2, \mathrm{CP}-\mathrm{D}$ 粘贴数为 2 , 且粘贴时以 0.5 的概率进行翻转操作. 实验结果 如表 6 所示, 其中第 1 列第 1 行对应实验 2 的最佳 实验结果.

第 1 部分和第 2 部分实验结果均表明，采用激 活型或抑制型的 CL 层都会大幅提升算法性能，但 激活型表现要好于抑制型. 同时发现，采用非线性 或线性 $\alpha$ 因子几乎没有区别. 表 5 实验结果表明,

\section{表 5 CL 层实验第 1 部分实验结果}

\begin{tabular}{lllllll}
\hline $\mathrm{CL}$ 层形式 & $\mathrm{AP} / \%$ & $\mathrm{AP}_{\mathrm{S}} / \%$ & $\mathrm{AP}_{\mathrm{M}} / \%$ & $\mathrm{AP}_{\mathrm{L}} / \%$ & $\begin{array}{c}\text { 训练 } \\
\text { 时长 } / \mathrm{h}\end{array}$ & $\begin{array}{c}\text { 推理 } \\
\text { 耗时 } / \mathrm{ms}\end{array}$ \\
\hline & 44.9 & 25.6 & 47.4 & 57.4 & & $401(340)$ \\
激活型 $+\alpha_{1}$ & $\mathbf{4 6 . 6}$ & $\mathbf{2 7 . 4}$ & $\mathbf{4 8 . 3}$ & $\mathbf{5 8 . 1}$ & $\mathbf{1 7 4 . 3}$ & $\mathbf{3 9 3}$ \\
激活型 $+\alpha_{2}$ & 46.5 & 27.5 & 48.1 & 58.2 & 174.0 & 398 \\
抑制型 $+\alpha_{1}$ & 45.8 & 26.8 & 47.9 & 57.9 & 174.2 & 402 \\
抑制型 $+\alpha_{2}$ & 45.8 & 26.9 & 47.7 & 58.0 & 174.9 & 395 \\
\hline
\end{tabular}

注. 粗体表示本实验最佳结果.

表 6 CL 层实验第 2 部分实验结果

\begin{tabular}{lcccccc}
\hline $\mathrm{CL}$ 层形式 & $\mathrm{AP} / \%$ & $\mathrm{AP}_{\mathrm{S}} / \%$ & $\mathrm{AP}_{\mathrm{M}} / \%$ & $\mathrm{AP}_{\mathrm{L}} / \%$ & $\begin{array}{c}\text { 训练 } \\
\text { 时长 } / \mathrm{h}\end{array}$ & $\begin{array}{c}\text { 推理 } \\
\text { 耗时 } / \mathrm{ms}\end{array}$ \\
\hline & 45.9 & 27.3 & 47.9 & 57.1 & 161.1 & 402 \\
激活型 $+\alpha_{1}$ & $\mathbf{4 7 . 4}$ & $\mathbf{2 8 . 3}$ & $\mathbf{4 9 . 1}$ & $\mathbf{5 8 . 7}$ & $\mathbf{1 7 4 . 5}$ & $\mathbf{3 9 6}$ \\
激活型 $+\alpha_{2}$ & 47.4 & 28.4 & 49.0 & 58.6 & 174.2 & 400 \\
抑制型 $+\alpha_{1}$ & 46.8 & 27.9 & 48.5 & 58.4 & 174.1 & 395 \\
抑制型 $+\alpha_{2}$ & 46.9 & 27.8 & 48.7 & 58.4 & 175.0 & 399 \\
\hline
\end{tabular}

注. 粗体表示本实验最佳结果.

单独使用 $\mathrm{CL}$ 层时, $\mathrm{AP}, \mathrm{AP}_{\mathrm{S}}, \mathrm{AP}_{\mathrm{M}}$ 和 $\mathrm{AP}_{\mathrm{L}}$ 依次分别 提升 $1.7 \% ， 1.8 \% ， 0.9 \%$ 和 $0.7 \%$. 将 $\mathrm{CP}$ 模块与 $\mathrm{CL}$ 层相结合得到了本文最佳实验结果, 将 CenterNet 单尺度检测 AP 从 $44.9 \%$ 提升到 $47.4 \%$, 提升了 $2.5 \%, \mathrm{AP}_{\mathrm{S}}, \mathrm{AP}_{\mathrm{M}}$ 和 $\mathrm{AP}_{\mathrm{L}}$ 分别提升了 $2.7 \%, 1.7 \%$ 和 $1.3 \%$. 还可以看到, CP 模块对小目标检测准确率 的提升是明显的, CL 层则在整体上进一步提升了 算法性能.

本文结合 CL 层机制来分析激活型与抑制型产 生差别的原因:

(1) 训练开始时， $\alpha$ 因子较大，此时采用抑制 型会大幅度缩小目标检测网络的搜索空间，当 $\alpha$ 
因子逐渐变小后，网络需要重新对受抑制的区域 进行搜索, 对网络训练造成影响.

(2) 采用激活型时，搜索区域没有缩小，网络 能够适应激活型带来的变化，在正常的搜索空间 内搜索正样本会产生较好的结果.

分析表 5 和表 6 中的训练时长数据可知，在使 用预训练模型情况下，嵌人 CL 层进行实验，训练 时长约为 $174 \mathrm{~h}$ ，相比仅使用 $\mathrm{CP}$ 模块，训练时长增 加了约 $14 \mathrm{~h}$ ，但 AP 提升了 $1.5 \%$ ，可见这些额外的 训练时长开销是值得的.

表中的推理耗时数据反映了硬件对检测速度 的直观影响. 原 CenterNet 使用一个 NVIDIA Tesla

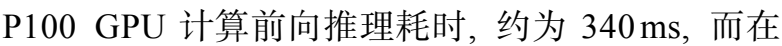
本文的硬件配置条件下，推理耗时约为 $400 \mathrm{~ms}$. 对 比数据可以看到, CP 模块和 CL 层都没有对检测速 度产生影响, 原因是 $\mathrm{CP}$ 模块是一种离线处理模块, 其不参与训练过程, 而 CL 层只在训练阶段嵌人网 络实现功能, 不参与网络前向推理阶段.

第 3 部分选用激活型 CL 层, 将其嵌人在 Hourglass 104 模块的第 2 个 Hourglass 52 模块最后 一层特征层上，不使用 $\mathrm{CP}$ 模块，选用非线性 $\alpha$ 因 子，与原 CenterNet 实验结果进行对比，验证 CL 层 的适用性. 实验结果如表 7 所示, 其中第 1 行第 1 列对应原 CenterNet 实验结果.

表 7 CL 层实验第 3 部分实验结果

\begin{tabular}{ccccc}
\hline $\mathrm{CL}$ 层形式 & $\mathrm{AP}$ & $\mathrm{AP}_{\mathrm{S}}$ & $\mathrm{AP}_{\mathrm{M}}$ & $\mathrm{AP}_{\mathrm{L}}$ \\
\hline & 44.9 & 25.6 & 47.4 & 57.4 \\
激活型 $+\alpha_{1}$ & $\mathbf{4 6 . 2}$ & $\mathbf{2 6 . 9}$ & $\mathbf{4 8 . 6}$ & $\mathbf{5 8 . 5}$ \\
\hline
\end{tabular}

注. 粗体表示本实验最佳结果.

结合表 5 和表 7 可以看到, 不使用 $\mathrm{CP}$ 模块，单 独将 CL 层嵌人到不同的特征层上, 可以不同程度 地提升算法性能，嵌人第 1 个 Hourglass52 模块使 $\mathrm{AP}$ 提高了 $1.7 \%$, 嵌人第 2 个 Hourglass 52 模块使 $\mathrm{AP}, \mathrm{AP}_{\mathrm{S}}, \mathrm{AP}_{\mathrm{M}}$ 和 $\mathrm{AP}_{\mathrm{L}}$ 依次提高了 $1.3 \%, 1.3 \%$, $1.2 \%$ 和 $1.1 \%$ ，这说明 CL 层嵌人位置选择具有一定 的适用性。

\section{4 结论与展望}

为解决目标检测网络在 COCO 2017 数据集上 小目标检测准确率低的问题, 本文从统计意义上 分析产生问题的原因，提出 $\mathrm{CP}$ 模块. 该模块采用 复制粘贴的思路，提高了包含小目标图片数量占
比和小目标面积占比, 同时增加了小目标平均 Anchor 匹配数量. 结合 CL 思想, 本文还提出 2 种 形式的 CL 层嵌人在目标检测网络特征提取层. 通 过一系列对比实验，证明其对 CenterNet 目标检测 性能提升效果是显著的.

$\mathrm{CP}$ 模块是一种离线处理模块，避免了多余的 网络训练开销, CL 层以嵌人的形式来使用, 不改 变网络整体结构, 仅在训练阶段开启, 而在推理阶 段关闭，不影响检测速度. 本文提出的 CL 层可以 用于不同的目标检测网络而不仅限于本文的使用 场景，存在特征提取层的网络都可以尝试使用以 提升网络性能. 同样, $\mathrm{CP}$ 模块也可以用于其他数 据集，以解决数据集各尺度目标分布不平衡等问 题. 本文方法仍有很大的扩展研究空间, CP 模块 和 CL 层存在进一步优化改进的可能, 这将作为未 来的研究内容.

\section{参考文献(References):}

[1] Liu L, Ouyang W L, Wang X G, et al. Deep learning for generic object detection: a survey[J]. International Journal of Computer Vision, 2020, 128(2): 261-318

[2] Zhou Z X, Shi Z W, Guo Y H, et al. Object detection in 20 years: a survey[OL]. [2020-05-24]. https://arxiv.org/abs/1905. 05055

[3] Yuan Gonglin, Hou Jing, Yin Kuiying. Night-time aerial image vehicle recognition technology based on transfer learning and image enhancement[J]. Journal of Computer-Aided Design \& Computer Graphics, 2019, 31(3): 467-473(in Chinese) (袁功霖, 侯静, 尹奎英. 基于迁移学习与图像增强的夜间 航拍车辆识别方法 [J]. 计算机辅助设计与图形学学报, 2019, 31(3): 467-473)

[4] Liu W, Anguelov D, Erhan D, et al. SSD: single shot multibox detector[C] //Proceedings of European Conference on Computer Vision. Heidelberg: Springer, 2016: 21-37

[5] Lin T Y, Goyal P, Girshick R, et al. Focal loss for dense object detection[C] //Proceedings of the IEEE International Conference on Computer Vision. Los Alamitos: IEEE Computer Society Press, 2017: 2999-3007

[6] Redmon J, Divvala S, Girshick R, et al. You only look once: unified, real-time object detection[C] //Proceedings of the IEEE Conference on Computer Vision and Pattern Recognition. Los Alamitos: IEEE Computer Society Press, 2016: 779-788

[7] Redmon J, Farhadi A. YOLO9000: better, faster, stronger[C] // Proceedings of the IEEE Conference on Computer Vision and Pattern Recognition. Los Alamitos: IEEE Computer Society Press, 2017: 6517-6525

[8] Redmon J, Farhadi A. YOLOv3: an incremental improvement[OL]. [2020-05-24]. https://arxiv.org/abs/1804.02767

[9] Lin T Y, Dollár P, Girshick R, et al. Feature pyramid networks for object detection[C] //Proceedings of the IEEE Conference on Computer Vision and Pattern Recognition. Los Alamitos: 
IEEE Computer Society Press, 2017: 936-944

[10] Lin T Y, Maire M, Belongie S, et al. Microsoft COCO: common objects in context[C] //Proceedings of European Conference on Computer Vision. Heidelberg: Springer, 2014: 740-755

[11] Law H, Deng J. CornerNet: detecting objects as paired keypoints[C] //Proceedings of European Conference on Computer Vision. Heidelberg: Springer, 2018: 765-781

[12] Duan K W, Bai S, Xie L X, et al. CenterNet: keypoint triplets for object detection[C] //Proceedings of the IEEE International Conference on Computer Vision. Los Alamitos: IEEE Computer Society Press, 2019: 6568-6577
[13] Newell A, Yang K Y, Deng J. Stacked hourglass networks for human pose estimation[C] //Proceedings of European Conference on Computer Vision. Heidelberg: Springer, 2016: 483-499

[14] Bengio Y, Louradour J, Collobert R, et al. Curriculum learning[C] //Proceedings of the 26th Annual International Conference on Machine Learning. New York: ACM Press, 2009: 41-48

[15] Zhou X Y, Zhuo J C, Krähenbühl P. Bottom-up object detection by grouping extreme and center points[C] //Proceedings of the IEEE Conference on Computer Vision and Pattern Recognition. Los Alamitos: IEEE Computer Society Press, 2019: 850-859 